\title{
Design and Analysis of Crowd Sized Estimation Techniques
}

\author{
Jugal Kishor Gupta \\ Research Scholar \\ Department of Computer Science and Engineering \\ IFTM University
}

\author{
S K Gupta \\ Assistant Professor \\ Department of Computer Science \& Engineering \\ Bundelkhand Institute of Engineering and \\ Technology
}

\begin{abstract}
This paper considers the different technique of estimation of crowd densities, an important part of the problem of automatic crowd monitoring and control. A new technique based on texture description of the images of the area under surveillance is proposed.
\end{abstract}

\section{Keywords}

crowd, image and surveillance etc.

\section{INTRODUCTION}

Security at venues, in particular cricket stadiums or other large scale area, where a large number of crowd tend to appear can be a critical business consideration. This is fited to surveillance systems using Closed Circuit Television (CCTV) where specific objects and their behaviour can be supervised through a long period of time. However, a human mind might be miss some essential data because superviousing crowds through CCTV is very difficult and require meor labour and cannot be operated for all the cameras simultaneously [10]. So , the need of automated techniques for superviousing crowds such as proximation of crowds strength ,location ,movement of crowd and observing crowd's behaviour, is required

\section{SURVEY}

A technique to estimate crowd densities have been proposed by Daviesetal[3], which has based on two measures removed from the input image of the area under surveillance. The first measure is the number of front picture elements calculated by deducting the input image from a position image containing no people. The second measure is the number of advantage picture elements of the image computed by an edge recognition followed by a diminishing operation.

Daviesetal proved that there existence of a linear relationship between the number of people present in the area under observation and the two measures, which were combined by a linear Kalman filter [3] into an "optimal" estimation of crowd density. Inspite of the success of this technique for approximating crowd density in areas containing reasonable amounts of people, it cannot be applied successfully in areas with high density crowds because the linear relationship does not hold when there are many covered people in the image.With the growth of economic development and increasing people's social interaction, the culture of large malls, stadium and places such as subway station is increasing more and more serious issue due to crowd congestion. As per the news agency reports: On February 27, 2013, LaoHeKou of Hubei province occurred a stampede caused by crowd of local elementary school, 12 students were injured, 5 students died. Therefore, if we would analyze the events in advance and predict the likelihood of the risk, then timely and effective measures could be aken to resuce the crowd [5]. It can effectively avoid the social security problem that caused by the high crowd solidity. According to the different feature extraction methods, crowd density estimation techniques can be divided into three categories: 1)estimation based on pixels statistical categories; 2)estimation based on texture analysis technique;3) estimation based on individual characteristics. For the first category, Davies, Chow et al proposed a approach ofusing foreground-pixels, which is based on the relationship between number of people and crowd density using a linear methods for estimating the density. TheHong Kong Chow et al proposed a neural network method by using a mix of global learning algorithm forthe crowd density. The method is mainly based on theextraction of the three characteristics of the crowd: population object edge length, a proportion of object pixels in the image and the background of the object pixels in the image. Paragios [1] proposed a method based on Markov Random Field (MRF) real-time crowd density estimation. The method is divided into two steps: The first step is to distinguish the image's foreground and background by Markov Random Field; The second step isto get the image by changing detection combined with a geometric module to perspective correction, and then estimate the population density of the monitored area. Chow et al. joined the body template matching module and match for human body for the people who are clearer individual characteristics and near camera [5].Jia Hong Yin eet.al proposed two technique to enhance crowd density measurement using areference image. A reference image with only backgroundis used to classify image pixels as belonging to eitherpedestrians or background so that a functionalrelationship between number of pedestrianclassifiedpixels and number of people can be established for crowddensity measurement. The automatic backgroundgenerator proposed exploits an intensity region related tothe average pixel intensity of each image to segmentbackground pixels and puts background pixels from asequence of crowd images together to obtain an artificialbackground image. The calibration approach, with whicha previously-established function for one site can be usedto estimate crowd density at various other sites, involves calibration of the image as opposed to calibration of thecamera. The results from two investigated sites using thebackground generator and the calibration have beenshown to be compatible with those from the site where thefunction between number of pedestrian-classified pixelsand number of people was established with a referenceimage taken from site. The techniques increase thegeneral applicability of the crowddensity estimationmethod developed by the authors. Both backgroundgeneration and calibration may be used in othersurveillance systems such as vehicle monitoring.[6]; 


\section{CROWD DENSITY}

Prof Dr. G. ketih the meaning of crowd density, In spaces of public assembly how it is important to differentiate that spaces are used. There are two types fundamentals - static and dynamic (places where the crowds perticularlly stand and spaces where the crowds are classically moving). The static and dynamic space will have different utillisation factors, So we do experience different densities and different crowd management requirements. For example a higher crowd densities nearby to the front of stage will be experienced by a concert. Routes to and from entry/exit points, bars, concession etc. it should be differentiated in the site plan and showed so that operators know where the crowds are moving and where crowds are perticularlly static. Onekey element tothis is evaluating the use (utilisation factor) of the site.

\subsection{Static Crowd Density}

The static crowd density and the impact of crowd density (the number of people per square metre) was still purposed by Prof Dr. G Keith for a standing crowd and a moving crowd, and it is important to understand for crowd security. For example, to evaluate the efficiency of crowd movement, amount and rates of fill for places of public get-together and the relative risks of both standing crowd density and the moving crowd density need to be understanded by you. The diagrams below show the standing density in people per square metre.

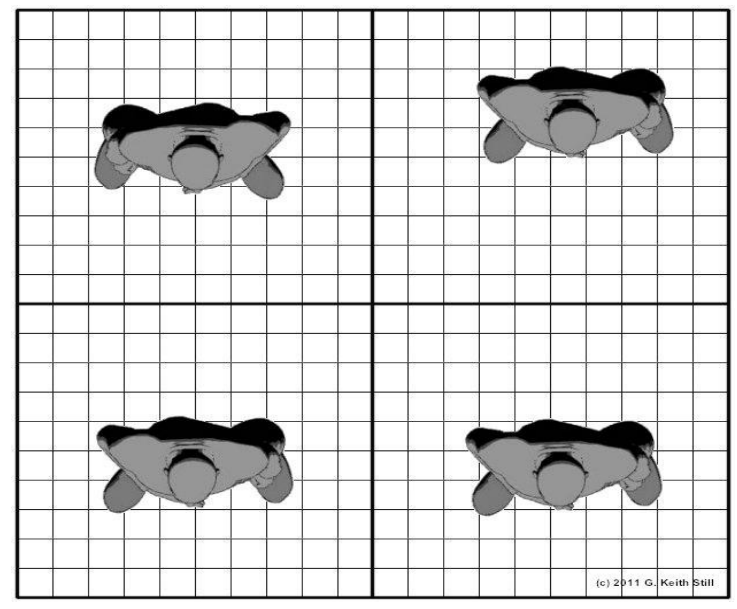

Figure 1: Person per square metre

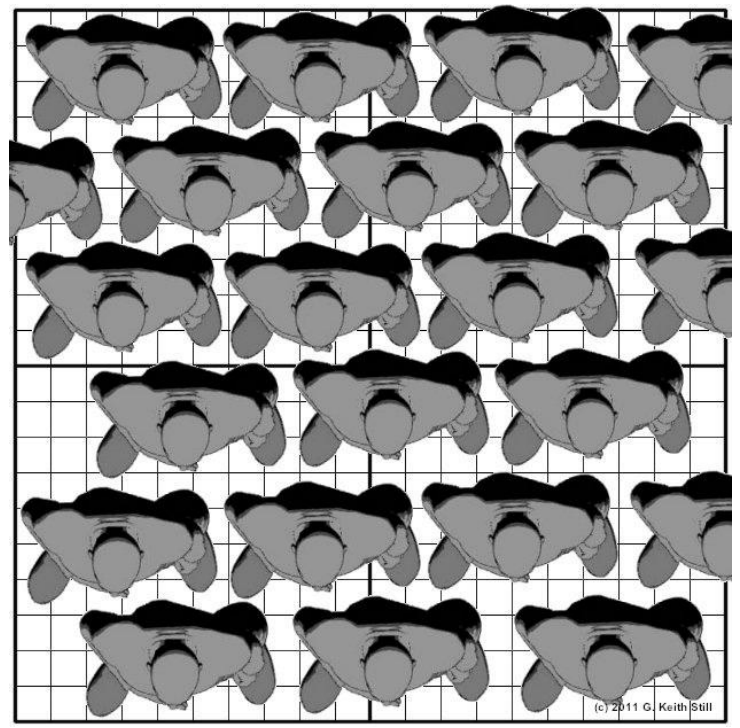

Figure 2 Person per square metre

\subsection{Visualising Crowd Density}

A 3D crowd visualiser for control room applications was developed by us. The $3 \mathrm{D}$ crowd visualiser allows us to both train staff to finding density from CCTV angles and to provide control room pictures for crowd build up during an event. Each grid is $5 \mathrm{~m}$ by $5 \mathrm{~m}$.

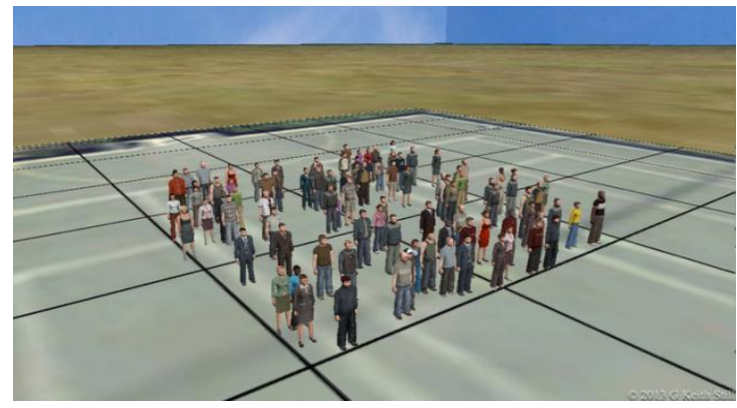

Figure 3 person per square metre

\section{WHY WE NEEED CALCULATION OF REAL CROWD DENSITIES?}

We have two reasons to tell about why we need figure out to crowd densities. Firstly, In Germany Government require a calculation of the maximum attendance limit in any government event. Secondly, At major events, the actual crowd densities are needed in order to be able to give information on crowd numbers and associated ordinary flows, as well as on how many first aid services will be required. Yet the Rather than implementing individual communication of people, this work make use of information at a large global level prepared by the crowd solidity and place geometry. We show that automatically acquired person solidity estimates can be applied to improv location of person and tracking performance in crowded scenes [4].

approach of these government event regulations is not to ask: 'how many people can a particular area hold?' rather: 'how many people can be contained without risk?', thus restricting the number of people to a completely harmless mass in terms of safety regulations. This is how the figure of $2 \mathrm{p} / \mathrm{m} 2$ was established in Germany. Projections in other countries are, to varying degrees, considerably higher or lower (5). If we examine crowd density values that present a clear risk, e.g. at bottlenecks or during evacuations, then a value of $6 \mathrm{p} / \mathrm{m} 2$ is often estimated in Germany. Measurements in Japan, however, show up to $8 \mathrm{P} / \mathrm{m} 2$

.How realistic or how useful is the designation of the number of people per $\mathrm{m}^{2}$ ? Can the number of people be used without taking into account the characteristics of the peoplethemselves?

\subsection{Mickel Theory}

Locating and detecting people in crowded area is a vital component for a large range of applications that including surveillance, group behavior personation and crowd distrees prevention. The dependable person detect and track in crowds, nevertheless, that is a highly challenging task cause of heavy occlusions, different viewpoint and varying solidity of people and the confusing appearance of body parts as well , e.g. the shoulder of one person could be similar to a head of a nearby person. High-soildity crowds present particular defiance due to the difficulty of secluded individual people with lowlevel standard methods of background subtraction and motion segmentation typically applied in low-soildiyu surveillance scenes [4]. 


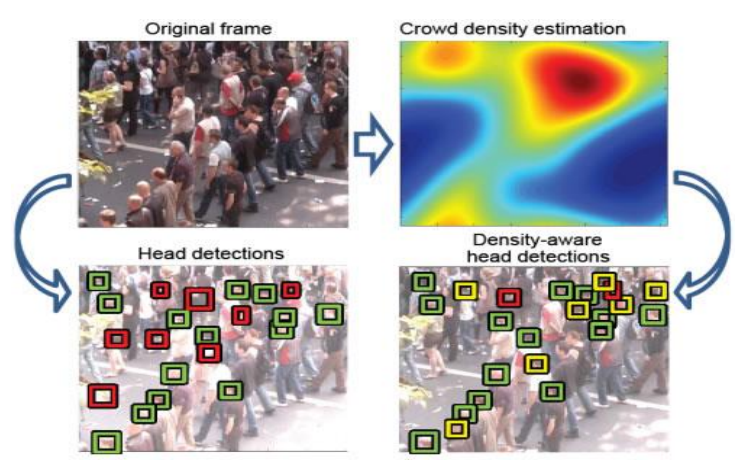

Figure 4: Overview of crowd density process [4]

\subsection{Methodology}

Flow chart of the estimation of crowd density with video.

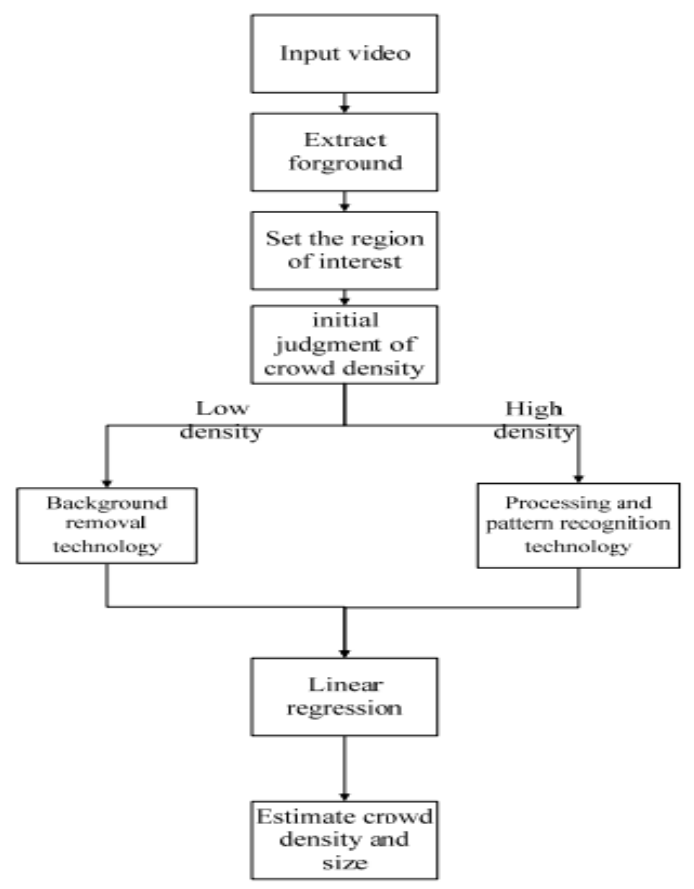

Figure 6: flow chart

\section{CONCLUSION}

In this survey We have discussed different techniques of crowd density estimation Techniques, which were discussed, are based on two measures extracted from the input image of the area under surveillance, "optimal" estimation of crowd density through a linear Kalman filter, estimation based on pixels statistical categories, estimation based on texture analysis technique, estimation based on individual characteristics.

\section{REFERENCE}

[1] Julio cezar Silvira Jacques Junior et.al , "crowd analysis using computer vision technique : A survey", IEEE signal Processing Magazine, September 2010.

[2] A.N.marana, S.A.Velastin, L.F.Costa, "Autommation Estimation of crowd density Using Texture", IWSIP'97,May 28-30.

[3] A.C. Davies, J.H. Yin, S.A. Velastin, "Crowd Monitoring using image processing", electronics And communications engineering journal\},February, pp. 3747, 1995.

[4] Mikel Rodriguez, Ivan Laptev, Josef Sivic, Jean-Yves Audibert, "Density-aware person detection and tracking in crowds",Proceedings of the IEEE International Conference on Computer Vision (2011), Poster.

[5] Bobo wang, hong bao, shan yang, and haitao lou, "crowd density estimation based on texture Feature extraction ",journal of multimedia, vol. 8, no. 4, august 2013 .

[6] Jia Hong Yin, sergio a. Velastin and anthony c. Davies, "image processing techniques forCrowd density estimation using a reference image",second asian conference on computer vision (accv95), singapore, 5-8 december, vol. Iii, pp 6-10.

[7] Qing WEN, Chengcheng JIA et al. People Number Estimation in the Crowded Scenes Using Texture Analysis Based on Gabor Filter. Journal of Computational Information Systems 7: 11 (2011) pp. 3754-3763

[8] Zhaoxiang Zhang, Min Li. Crowd density estimation based on statistical analysis of local intra-crowd motions for public area surveillance. Optical Engineering. 51(4), 2012

[9] D. B. Yang, H. H. Gonzalez-Banos, L. J. Guibas. Counting people in crowds with a real-time network of simple image sensors. Proceedings. Ninth IEEE International Conference on Computer Vision, 2003, 1 pp. $122-129$

[10] Kumar, T. and K. Verma, 2010a. A theory based on conversion of RGB image to gray image. Int. J. Comput. Appli., 7: 5-12. DOI: 10.5120/1140-1493

[11] S. Bouchafa, d. Aubert, and s. Bouza, "crowd motion Estimation and motionless detection in subway corridors By image processing", (itsc) intelligent transportation System, ieee conference, pp. 332 - 337, (1997). 